\title{
Experimental and computational study of tones occurring with a coaxial nozzle
}

\author{
K.B.M.Q. Zaman ${ }^{1}$, I. Milanovic ${ }^{2}$, A.F. Fagan ${ }^{3}$ and C.J. Miller ${ }^{4}$
}

\begin{abstract}
The source of audible tones occurring with a coaxial nozzle in a range of low Mach numbers is explored experimentally as well as computationally. The hardware is comprised of an inner and an outer nozzle, without a center-body, that are held together by a set of four struts. With increasing jet Mach number $\left(M_{j}\right)$, first a tone occurred at about $2550 \mathrm{~Hz}$ around $M_{j}=0.06$. At higher values of $M_{j}$ a tone at $5200 \mathrm{~Hz}$ dominated the noise spectra. The corresponding nondimensional frequency, based on effective thickness of the inner nozzle lip and jet exit velocity, turned out to be about 0.2 , a value characteristic of Karmann vortex shedding. Thus, vortex shedding from the inner nozzle lip could be linked to the tones. From a comparison of the acoustic wavelengths and the nozzle dimensions, it was inferred that the vortex shedding excited a one-quarter-wave resonance within the divergent section of either the inner nozzle or the outer nozzle. This led to the generation of the sharp tones.
\end{abstract}

\section{Introduction}

The subject addressed in this paper is certain 'spurious' tones occurring with nozzle flows. Many previous studies have addressed tones and resonances occurring with jets and shear layers and their interactions with structural elements in the vicinity of the flow [e.g., 2-6]. Apart from the wellknown phenomena such as, edgetones, cavity resonance, screech etc., sometimes tones are encountered especially at low subsonic conditions. As the flow velocity of a nozzle is increased from zero, distinct tones may occur. With increasing speed a tone may disappear and new ones with different frequencies may appear. These tones, of unknown origin, are often ignored as long as they are not audible at the operating condition.

\footnotetext{
${ }^{1}$ Inlets \& Nozzles Branch, NASA GRC, AIAA Associate Fellow.

${ }^{2}$ University of Hartford, West Hartford, CT 06117, Department of Mechanical Engineering, AIAA Senior Member.

${ }^{3}$ Optics \& Photonics Branch, NASA GRC, AIAA Associate Fellow.

${ }^{4}$ Acoustics Branch, NASA GRC, AIAA Senior Member.
} 
The tone problem appears to get exacerbated with coaxial, multiple-stream nozzles. The geometry of such a nozzle typically involves two or more coannular ducts as well as a center-body. Usually, three or four struts, spaced equally in the circumferential direction, hold these coannular elements together. The present study is actually a follow-up of the work reported in Ref. [1]. As an introduction to the problem at hand, key results from [1] are reviewed in the following. For a specific coannular nozzle, it was shown in [1] that the origin of some prominent tones was linked to "vortex shedding' from the struts. Here 'vortex shedding' refers to the well-known asymmetric Karmann vortex street phenomenon in a wake, and the terms 'vortex shedding', 'Strouhal shedding' or just 'shedding' will be used synonymously in the following. The shedding coupled with acoustic resonance modes of the interior of the nozzle. The result was an acoustic tone that was spectrally more concentrated than that from vortex shedding alone. Note that such coupling has long been known to produce resonances in various other flow configurations. For example, 'Parker modes' refer to such a phenomenon occurring with a cascade of blades when placed in a wind tunnel [7].

1.1 Summary of Ref. [1]: The investigation reported in [1] was prompted by objectionable tones occurring in large-scale tests involving a three-stream as well as a two-stream nozzle at NASA Glenn Research Center (GRC), [8]. In the two-stream case, a sharp $7 \mathrm{kHz}$ tone, occurring at high subsonic conditions, persisted in spite of extensive efforts aimed at its mitigation. A half-scale model of this nozzle was then fabricated to investigate the phenomenon in a smaller facility where parametric variations could be done in a relatively easy and cost-effective manner. The primary objective was to understand the origin of the tones and find possible remedies. Figure 1(a), reproduced from [1], shows a schematic diagram of the model-scale nozzle, while Fig. 1(b) shows a picture with view from the upstream end. A set of four struts held the outer and inner nozzles together with the center-body. Flow from the same supply was split and routed through the inner and outer passages. For the given geometry, with 'untreated' struts, distinct tones occurred in the range, $0.12<M_{j}$ $<0.43$; (the jet Mach number $M_{j}$ is defined in section 2). With increasing $M_{j}$ the frequency remained approximately a constant in a 'stage' then jumped to a higher frequency in the next stage, there being four distinct stages. It was observed that the tone frequency in each stage, when nondimensionalized by the strut thickness and the local mean velocity, corresponded to a Strouhal number of about 0.2. Furthermore, the occurrence of the tones shifted to a higher Mach number range when the strut thickness was increased, to be commensurate with the 0.2 value of Strouhal 
number. With strut thickness increased from 0.32 to $0.66 \mathrm{~cm}$, the $M_{j}$ range where sharp tones occurred shifted from $0.12-0.43$ to $0.4-0.85$; the tones in the higher $M_{j}$ range were also much louder.

These observations led to the inference that vortex shedding from the struts was an essential part of the mechanism for the generation of the tones. The fact that the frequency varied in stages and remained constant within a stage indicated that there was a 'locking on', possibly with duct acoustic modes. These inferences were further substantiated by the fact that the tones disappeared when small caps (Fig. 1b) were attached to the struts. Apparently, the two-dimensionality of the flow over the struts was broken, and thus vortex shedding was disrupted, resulting in an elimination of the tones.

In spite of the understanding advanced by the experiments, the nature of the duct acoustic modes that locked on to the vortex shedding remained far from being clear. To that end a numerical study was conducted subsequently. A perturbation was imparted near the trailing edge of one of the struts simulating the asymmetric vortex shedding. For a given perturbation frequency and amplitude the unsteady pressure field in the entire nozzle and its vicinity was computed. Frequency sweeps yielded resonant conditions at practically the same frequencies as noted in the experiment. For each resonant frequency the computed pressure distribution exhibited standing waves. The nature of the standing waves ('mode shapes') associated with the resonances (tones) were then examined.

As an example, the mode shape for the fundamental tone (stage 1, at $4.5 \mathrm{kHz}$ ) is shown in Fig. 1(c) with four iso-surfaces of the instantaneous pressure amplitude. At a given cross-sectional plane, an asymmetric pressure field is observed. Maximum amplitudes occur in the intra-strut spaces. While the perturbation is imparted at only one strut, it is noted that comparable pressure amplitudes occur at each of the other three struts, indicating a synchronized motion under the resonance. From Fig. 1(c) it is evident that the pressure distribution is not characteristic of a simple plane-wave, helical or radial mode shape. It is quite complex. At higher resonant frequencies even more complex shapes took place. The numerical study also revealed that there could be numerous such acoustic modes, however, only a limited number of them could be excited by the asymmetric perturbation from the struts. Further details of the numerical method and the results should become clearer when similar 
results for the coaxial nozzle under current study are described in the following. A curious reader may also find more details of the numerical procedures in Ref. [9].

1.2 Motivation and objective: The present study was motivated by the observations in another past experiment [10] that addressed the effect of shock waves on the development of a jet. A coaxial nozzle was used to study the effect of the shocks produced by a wedge in the outer stream on the development of the inner jet. The nozzle did not have a center-body. The inner and outer ducts were held together by four struts. While running those experiments tones were also heard at low Mach numbers. As observed in [1], with increasing $M_{j}$ the tones went through a staging behavior. These tones remained unexplained and were ignored at the time since the focus was on supersonic flows. After the results of [1] were obtained, of curiosity was the question if vortex shedding from the struts also instigated the tones for this nozzle. This was soon ruled out, as it will be elaborated in the following. Further experiments were then conducted followed by a numerical study in order to obtain an understanding of the mechanisms involved. These results are presented in this paper.

\section{Experimental Facility}

The experiments are conducted in an open jet facility at NASA GRC, a description of which can be found in prior publications (e.g., [1], [10]). The nozzle, shown by the picture in Fig. 2(a) is the same one as used in [10]. It is comprised of an inner nozzle with $1.40 \mathrm{~cm}$ exit diameter and an outer nozzle with $5.08 \mathrm{~cm}$ exit diameter $\left(D_{o}\right)$. There is no center-body and the two nozzles are held together by a set of four struts placed in a crossed shape. The struts, about $2 \mathrm{~cm}$ long in the streamwise direction, are made from $3.18 \mathrm{~mm}$ thick bar stock and have pointed edges in the direction of the flow. Each nozzle has convergent-divergent shape with nominal design Mach numbers of 1.6 and 1.4 for the inner nozzle and the outer nozzle, respectively. All data presented here, however, pertain to low Mach numbers at subsonic conditions. A schematic of the interior of the nozzle is shown in Fig. 2(b). The throat of the inner nozzle $(d=1.27 \mathrm{~cm})$ is located $1.689 \mathrm{~cm}$ upstream of the exit; the lip thickness is $1 \mathrm{~mm}$. The throat of the outer nozzle is located about $4.2 \mathrm{~cm}$ upstream of the exit (the throat is rounded over a distance contained by the two discs seen in the region marked by the arrow in Fig. 2b). The exits of the two nozzles are located at the same plane (coplanar). The coordinate system is indicated in Fig. 2(b), with the origin on the axis and at the entrance of the nozzle. The length of the nozzle is $0.1905 \mathrm{~m}$; the inner nozzle is of the same length. Thus, the exit is at 
$x=0.1905 \mathrm{~m}$, the throat of the inner nozzle is at $x=0.1736 \mathrm{~m}$ and that of the outer nozzle is at $x=0.1485 \mathrm{~m}$. Other pictures in Fig. 2 pertain to various diagnostic procedures tried in an attempt to identify the source of the tones; these will be described further in the following.

The same flow from the plenum chamber splits and passes through the inner nozzle and the outer nozzle (Fig. 2b). Thus, the 'jet Mach number' $\left(M_{j}\right)$ is approximately the same at the exits of the two. This is confirmed by a Pitot probe survey. The 'jet Mach number' is the Mach number had the flow expanded fully for a given pressure ratio and is given by, $M_{j}=\left(\left(\left(p_{0} / p_{a}\right)^{(\gamma-1) / \gamma}-1\right) \frac{2}{\gamma-1}\right)^{1 / 2}$, where, $p_{0}$ and $p_{a}$ are plenum pressure and ambient pressure, respectively. All data are for cold flow, i.e., having a total temperature the same everywhere as in the ambient.

Sound pressure level spectra are obtained using overhead microphones. The data presented in the following pertain to very low values of $M_{j}$. Instead of 'central air supply' that involves valve noise, an auxiliary blower is used to run these experiments. Nonetheless, the noise measurements are susceptible to small changes in the background noise (such as, from air conditioner, from supply lines when other facilities draw air, etc.). Care is taken to conduct all comparative measurements back-to-back. However, this should explain some differences in the broadband levels from figure to figure when those data are obtained on different days. Note that the focus here is on the sharp tones and their frequencies and the facility is considered adequate for those measurements. All spectral data shown in the following pertain to a microphone location at about $25 D_{o}$ from the axis and at $90^{\circ}$ polar location. The data acquisition is done using Labview® software. The spectrum analysis is done over 0-50kHz with a bandwidth of $50 \mathrm{~Hz}$.

\section{Results}

3.1 Experimental Results: Figure 3 shows sound pressure level spectra measured at different $M_{j}$. Successive traces are staggered by one major ordinate division. A broad peak can be noticed for $M_{j}$ $>0.126$ whose frequency continuously increases with increasing $M_{j}$ (e.g., about $22 \mathrm{kHz}$ at $M_{j}$ $=0.317$ ). This is due to vortex shedding from the lip of the inner nozzle, as can be inferred from the non-dimensional frequency (Strouhal number) based on the lip thickness $(1 \mathrm{~mm})$ and the jet exit velocity; (for the cited condition this turns out to be about 0.21 ). These broad spectral peaks are not 
accompanied by audible tones and are not the focus of the paper. However, they play an essential role in exciting the tones, as discussed shortly. It can be seen that at lower $M_{j}$ the spectra are characterized by sharp peaks. This is shown more clearly with expanded scales in Fig. 4. The sharp peaks are accompanied by audible tones. Note that there are two dominant peaks at about 2550 and 5200 $\mathrm{Hz}$, marked by the dashed vertical lines. The origin of these tones is pursued in the following.

Estimates of non-dimensional frequency indicate that vortex shedding from the struts has no bearing on the observed tones. Take for example the tone at $5200 \mathrm{~Hz}$ occurring at $M_{j}=0.105$ (Fig. 4). The Strouhal number based on the strut thickness $(3.2 \mathrm{~mm})$ and duct velocity near the location of the struts (about 3.87 times lower than the jet velocity, as estimated from continuity and pertinent crosssectional areas of the nozzle) turns out to be 1.78. This is far removed from the Strouhal shedding number of 0.2. Furthermore, application of 'caps' on the struts (see the picture of the back of the nozzle in Fig. 2c) did not have any effect on the spectra at all. This is shown by the noise spectra in Fig. 5 for three values of $M_{j}$,. These spectral data are presented in a log scale in order to cover a wider frequency range. Note that Strouhal shedding from the struts was the driving mechanism for the tones in [1]; thus, caps on struts effectively eliminated the tones. Here, shedding from the struts appears irrelevant to the origin of the tones noted in Figs. 3 and 4.

On the other hand, the same frequency of $5200 \mathrm{~Hz}$ corresponds to a Strouhal number of 0.15 when based on the inner nozzle lip thickness and the jet exit velocity. Similarly, the $2550 \mathrm{~Hz}$ at the lowest $M_{j}$ yields a Strouhal number of about 0.13 . Noting that the characteristic length scale should be larger than just the lip thickness due to boundary layer effects, these numbers are considered close enough to the Strouhal shedding value of 0.2 . These considerations suggest a role of the vortex shedding from the inner nozzle lip in the generation of the tones.

In order to investigate the link between vortex shedding from the lip and the tones, the response to two triangular tabs affixed to the nozzle lip (Fig. 2d) was explored. Their effect on SPL spectra are shown in Fig. 6 for the same three $M_{j}$ values as in Fig. 5. The spectral peaks are affected in amplitudes as well as frequency. However, they are not completely eliminated. The tabs were supposed to break the 'two-dimensionality' of the wake from the lip and thereby suppress the vortex shedding 
and hence the tones. Obviously, the two tabs failed to achieve that completely and shedding from the rest of the nozzle lip must have been enough to instigate the still persisting tones.

An attempt was made to clarify if the tones were tied specifically to either the inner or the outer stream. For this purpose the inner jet was blocked by covering the entrance with a plug and adhesive tapes. Figure 7 shows that blocking the inner nozzle took out the tones. Thus, the origin of the tones appeared to be linked to the inner nozzle flow. However, an inner flow with velocity comparable to that of the outer flow was necessary to produce a wake from the lip that would support the vortex shedding. Thus, it was no surprise in retrospect that without the inner flow there was no wake, hence no Strouhal shedding and therefore no tones.

By this time simple estimates led to a conjecture that the tones might be due to standing waves set up within the divergent sections of the nozzles, instigated by the shedding from the lip. The notion stemmed from the observation that the throat-to-exit distance of the inner nozzle corresponded to $1 / 4^{\text {th }}$ of the acoustic wavelength at $5200 \mathrm{~Hz}$. The mechanism of the standing waves in the divergent section was thought to be somewhat similar to that observed with the phenomenon of transonic resonance with C-D nozzles [11]. There, an unsteady shock near the throat excited the 1/4-wave resonance. In the present case, it is the shedding from the lip that apparently excited the $1 / 4$-wave resonance. This is explored further in the next subsection.

The tones in the present case could be eliminated by the placement of epoxy beads near the throat of the inner nozzle. This is shown in Fig. 8. Four epoxy beads were applied near the throat and these can be seen in Fig. 2e upon a close inspection. Not having a clear knowledge about the mechanism of the tones, the boundary layer distortion by the beads was tried since the same technique had successfully suppressed the transonic tones [11]. It is now realized that the tone mechanism here is not quite the same as in [11] and possible reasons why the beads eliminated the tones is discussed at the end of section 3.3. Referring back to section 2, note that the noise spectra at these low Mach numbers were susceptible to extraneous noise sources. This accounts for a difference in the baseline data (red curves) in Fig. 8 compared to those in Figs. 5-7. Data in Fig. 8 were taken on a different day. However, the blue and red curves in each figure were acquired back-to-back and thus the relative effects of the various devices should be valid. 
Before parting, a comment is in order regarding the Mach number range where the tones are heard. Here, the tones are audible in a very low $M_{j}$ range (about 0.05-0.12). However, recall the discussion of Ref. [1] in the Introduction. The Mach number range where the tones become prominent depends on pertinent dimensions. The range would be higher with a thicker inner nozzle lip as well as shorter throat-to-exit length of the nozzles. By the same token, these tones will not be a problem if the inner nozzle had a thin knife edge at the exit. The vortex shedding frequency might be too high and not compatible with the resonance of the divergent section.

3.2 Numerical simulation: In an effort to obtain a clearer understanding of the mechanisms associated with the tones, a numerical study is conducted using COMSOL multiphysics ${ }^{\circledR}$ software, similarly as done in [1]. The simulation is carried out for full 3-D geometry of the current nozzle, without flow. The computational domain starts upstream of the internal flow split between the primary and the outer flow paths, and extends downstream by about one $D_{o}$ from the exit (Fig. 2b). For a given perturbation technique, explained in the following, the simulation solves for the acoustic pressure and velocity distributions within the computational domain using a finite element formulation on an unstructured mesh. The upstream boundary has a plane wave radiation condition. A spherical wave radiation condition is used for the downstream boundary. The methods used are basically the same as followed in [1] and an interested reader may look up that reference as well as [9] for further details.

Different excitation schemes are tried. Figure 9 shows a schematic of the inner nozzle with the struts, having the outer nozzle removed. In one run of the simulation, two small panels on either side of one of the struts, colored pink and blue, are used to provide periodic perturbation. The panels are near the trailing edge and driven periodically with $\pm 1 \mathrm{~Pa}$, with opposite phase. In another run, the excitation is imparted from the lip of the inner nozzle (colored red in Fig.9). The amplitude was again $\pm 1 \mathrm{~Pa}$. The former excitation resembled a dipole source simulating the asymmetric vortex shedding, whereas the latter was like a monopole with the entire circular ring of the lip surface as being the source. Frequency sweep results for the two methods are shown in Fig. 10. The ordinate is the average of the absolute pressure integrated over the entire computational domain; the abscissa is the frequency of the excitation. As stated before, the 'strut panel' excitation in [1] had produced 
resonant peaks at the same frequencies as observed in corresponding experiments. Here, for the current nozzle, the strut panel excitation has not produced any prominent peak in the spectrum (blue curve at the bottom of Fig. 10). On the other hand, the 'inner lip' excitation has generated clear spikes in the spectrum. The frequencies of the dominant spectral peaks (2579 and $5200 \mathrm{~Hz}$ ) agree very well with the experimental observations of 2550 and $5200 \mathrm{~Hz}$ (Fig. 4). (There is a discontinuity in the spectrum around $4 \mathrm{kHz}$; this is because data from two separate sweeps at 1-4 kHz and 4-6 $\mathrm{kHz}$ had to be patched. A single sweep over 1-6 kHz failed due to software limitations.)

The instantaneous pressure $(p)$ distribution just downstream of the struts corresponding to the first dominant peak in the spectrum $(2579 \mathrm{~Hz}$, Fig. 10) is shown in Fig. 11. These data are for a given phase within the period of the excitation. The pressure distribution is found to be axisymmetric over the cross-section. Note that the corresponding distribution in [1] exhibited alternating positive and negative pressure cells in the intra-strut spaces (this can be gleaned from an inspection of Fig. 1c).

Iso-surfaces of instantaneous pressure corresponding to the spectral peak at $2579 \mathrm{~Hz}$ are shown in Fig. 12. In Fig. 12(a) data only in the outer nozzle are shown while those for both inner and outer nozzles are shown in Fig. 12(b). It becomes amply clear that the tone is accompanied by the presence of longitudinal standing waves. The distance between the blue iso-surfaces in the outer nozzle and that between, say, two red iso-surfaces in the inner nozzle are found to be the same, indicating that the wavelengths are the same in either nozzle. The wavelength is clearly obtained from the centerline pressure distribution shown in Fig. 13. For reference, in Fig. 13 the nozzle exit, the throat of the inner nozzle and the throat of the outer nozzle, located at $0.1905 \mathrm{~m}, 0.1736 \mathrm{~m}$ and $0.1485 \mathrm{~m}$, are indicated by the vertical lines. Note that the pressure plotted in Fig. 13 is instantaneous, i.e., at a particular phase of the excitation cycle. Thus, with varying phase the pressure distribution will go through changes in space and time, however, the wavelength should remain unchanged. The measured wavelength of $0.137 \mathrm{~m}$ is the same as that of an acoustic wave of frequency $2579 \mathrm{~Hz}$ at these low Mach numbers. One-quarter of this $(0.035 \mathrm{~m})$ roughly corresponds to the throat-to-exit distance in the outer nozzle $(0.042 \mathrm{~m})$. The two numbers may be close enough to suggest a link. It is plausible that a $1 / 4$-wave resonance may have been excited within the divergent section of the outer nozzle. This is further discussed in the next subsection. 
Similarly, the $5200 \mathrm{~Hz}$ tone may be linked to a $1 / 4$-wave resonance in the divergent section of the inner nozzle. The centerline pressure distribution for this case is shown in Fig. 14. The wavelength measured from this plot is $0.0675 \mathrm{~m}$. One quarter of this $(0.0169 \mathrm{~m})$ matches almost perfectly with the throat-to-exit length of the divergent section of the inner nozzle.

3.3 Further discussion of likely mechanism: Earlier, shedding from the struts was ruled out to be the 'driving' mechanism instigating the tones. Instead it became clear that the tones were linked to the shedding from the inner nozzle lip. Obviously, the shedding locked on to some acoustic modes to yield the sharp tones. The question remained as to what was the nature of those acoustic modes.

From the iso-surface patterns for $2579 \mathrm{~Hz}$ seen in Fig. 12 (or corresponding data for $5200 \mathrm{~Hz}$, having a similar appearance, and not shown for brevity), one might wonder if longitudinal 'duct modes' were being excited by the lip shedding to cause the tones. Note that the iso-surfaces in the inner nozzle (Fig. 12b) are of much larger amplitudes than those in the outer nozzle. Now, consider the inner nozzle which is $0.1905 \mathrm{~m}$ long. Assuming a half-wave resonance in a duct with both ends open, a fundamental frequency of about $900 \mathrm{~Hz}$ would be expected. The spectral peak with the lowest frequency in the present case is $2579 \mathrm{~Hz}$. For the sake of argument let us assume that the latter frequency is the third harmonic of a fundamental at $900 \mathrm{~Hz}$. However, if that were the case other harmonics of $900 \mathrm{~Hz}$ would also be expected to appear in the spectra. For example, between 2579 $\mathrm{Hz}$ and $5200 \mathrm{~Hz}$ two more peaks would be expected representing the $4^{\text {th }}$ and $5^{\text {th }}$ harmonics. In most cases, the spectra do not exhibit such peaks. It leaves little doubt that $2579 \mathrm{~Hz}$ is the fundamental frequency of the phenomenon under consideration. Thus, longitudinal duct modes may be ruled out from the present consideration.

There does not appear to be any other plausible duct modes that would produce the standing waves as seen in Fig. 12-14. This leaves us with the 1/4-wave resonance within the divergent sections as the most plausible 'carrier' mechanism that locks on to the lip shedding to generate the tones. This led to the postulation made in sections 3.1 and 3.2. 
The postulation is supported by the matching of the one-quarter-wavelength with the throat-to-exit distance. Recall that this matching for the lower frequency case $(2579 \mathrm{~Hz})$ is rather approximate. On the other hand, the matching for the $5200 \mathrm{~Hz}$ case is very good. The postulation invokes two separate sources for the two frequencies, viz., the divergent section of the outer nozzle responsible for the $2579 \mathrm{~Hz}$ tone and the divergent section of the inner duct for the $5200 \mathrm{~Hz}$ tone. The two frequencies $2579 \mathrm{~Hz}$ and $5200 \mathrm{~Hz}$, however, are harmonically related within the frequency resolutions of the analyses as well as the measurements. It is not clear if this is just a coincidence or because the lengths of the two divergent sections are related approximately by a factor of two. It is possible there is an interaction and the two are excited simultaneously. In any case, the lip shedding exciting a $1 / 4$-wave resonance in the divergent sections of the inner and the outer nozzles appears to be the best possible explanation for the generation of the tones.

With reference to Fig. 3, once again, it may be noted that the spectral peaks due to Strouhal shedding from the inner nozzle lip become concentrated into sharp spikes as the jet Mach number is reduced $\left(M_{j}<0.126\right)$. The frequency of shedding at low $M_{j}$ matches the frequency of one-quarter-wave resonance within the divergent sections of the nozzles. There is a lock-in that gives rise to the sharp spectral peaks and the accompanying audible tones.

It is worth noting that the one-quarter-wave resonance is an axisymmetric (plane-wave) phenomenon whereas the vortex shedding from the lip of the inner nozzle is an asymmetric phenomenon when viewed across the inner nozzle wall. However, it involves alternate vortex rings of opposite sense shed in the inner and outer nozzles both at the same frequency ( $180^{\circ}$ out of phase). When only the flow in the inner nozzle is considered it experiences the passage of symmetric vortex rings providing an axisymmetric perturbation. Similarly, the outer nozzle also experiences a symmetric perturbation from the passage of the outer vortex rings. Thus, the one-quarter-wave resonance for either the inner or the outer nozzle is excited when the shedding frequency is appropriate.

Finally, recall the effect of the epoxy beads near the inner nozzle throat that suppressed the tones. It is thought this was simply due to boundary layer distortions that interrupted the lip shedding. The four epoxy beads introduced distortions in the boundary layer entering the divergent section. These distortions grew as they reached the exit of the nozzle. The distorted flow apparently broke the 
'two-dimensionality' of the wake from the lip - more than that achieved by the two tabs (Fig. 6) and thus caused a complete elimination of the tones (Fig. 8).

\section{Conclusions}

The coaxial nozzle under consideration emits tones at $2579 \mathrm{~Hz}$ and $5200 \mathrm{~Hz}$ in a range of low Mach numbers. These frequencies are found to be far removed from the expected vortex shedding frequencies in the wake of the struts. Thus, vortex shedding from the struts being the instigator of the tones is ruled out. Instead, the spectral peak frequencies corresponded to the Strouhal number of vortex shedding from the lip of the inner nozzle. From these results and geometric considerations it is conjectured that the shedding must lock-on to a one-quarter-wave resonance in the divergent sections of either the inner or the outer nozzle leading to the generation of the tones. This is supported by numerical simulation. First, the simulation, upon a frequency sweep and with perturbation on the inner nozzle lip, is found to produce resonances at identical frequencies as noted in the experiment. Then, an inspection of the longitudinal pressure distributions confirm that one-quarter of the wavelengths correspond to the throat-to-exit length of the divergent section of either the outer nozzle (for $2579 \mathrm{~Hz}$ ) or the inner nozzle (for $5200 \mathrm{~Hz}$ ).

\section{Acknowledgement}

Support from the Transformational Tools and Technologies (TTT) Project as well as the Commercial Supersonics Technologies (CST) Project of NASA’s Advanced Air Vehicles Program is gratefully acknowledged. The second author is grateful to NASA GRC Faculty Fellowship Program for supporting a summer visit to NASA GRC in 2017 when much of the numerical simulation was done.

\section{References:}

[1] Zaman, K. B. M. Q., Bridges, J.E., Fagan, A.F. and Miller, C.J., "Tones Encountered with a Coannular Nozzle and a Method for Their Suppression”, AIAA J., January, 2018, DOI: 10.2514/1.J056676

[2] Rockwell, D. and Naudascher, E., "Self-sustained oscillations of impinging shear layers", Ann. Rev. of Fluid Mech., vol. 11, pp. 67-93, 1979.

[3] Nomoto, H. and Culick, F.E.C., "An experimental investigation of pure tone generation by vortex shedding in a duct”, J. Sound \& Vib., vol. 84, issue 2, pp. 247-252, 1982.

[4] Raman, G. and Srinivasan, K., "Whistles: from toys to industrial devices and applications", Noise Notes, vol. 8, no. 3, July, 2009. 
[5] Topalian, V.D. and Freund, J.B., “Acoustic resonance in a model ducted-jet system”, AIAA J., vol. 48, no. 7, pp. 1348-1360, 2010.

[6] Zaman, K.B.M.Q., Clem, M.M. and Fagan, A.F., "Noise from a jet discharged into a duct and its suppression”, Int. Journal of Aeroacoustics, vol. 12, no. 3, pp. 189-214, 2013.

[6] Henderson, B.H. and Huff, D.L., The Aeroacoustics of Offset Three-Stream Jets for Future Commercial Supersonic Aircraft, AIAA Paper 2016-2992, 22nd AIAA/CEAS Aeroacoustics Conference, 30 May - 1 June, 2016, Lyon, France.

[7] Parker, R. and Griffiths, W.R., "Low frequency resonance effects in wake shedding from parallel plates", J. Sound and Vib., vol. 7, no. 3, pp. 371-379, 1968.

[8] Henderson, B.H. and Huff, D.L., The Aeroacoustics of Offset Three-Stream Jets for Future Commercial Supersonic Aircraft, AIAA Paper 2016-2992, 22nd AIAA/CEAS Aeroacoustics Conference, 30 May - 1 June, 2016, Lyon, France.

[9] Milanovic, I., Zaman, K.B.M.Q. and Miller, C.J., 2018, "Resonance and tones in dual-stream nozzles induced by vortex shedding from struts", submitted for presentation at the Aviation Forum, June 25-29, 2018, Atlanta, GA.

[10] Castner, R.S., Zaman, K.B.M.Q., Fagan, A.F. and Heath, C.M., 2017, “Wedge shock and nozzle exhaust plume interaction in a supersonic jet flow", J. of Aircraft, Vol. 54, No. 1, pp. 125-134.

[11] Zaman, K.B.M.Q., Dahl, M.D., Bencic, T.J. and Loh, C.Y., 2002, "Investigation of a 'transonic resonance' with convergent-divergent nozzles", J. Fluid Mech., 463, pp. 313-343 


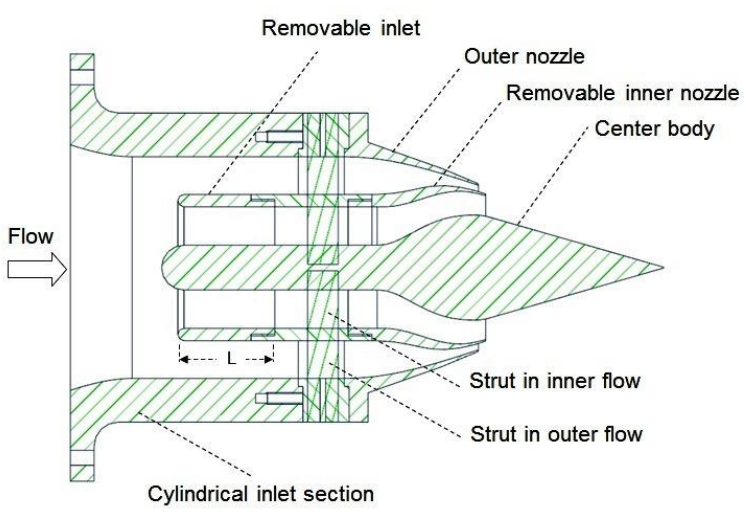

(a)

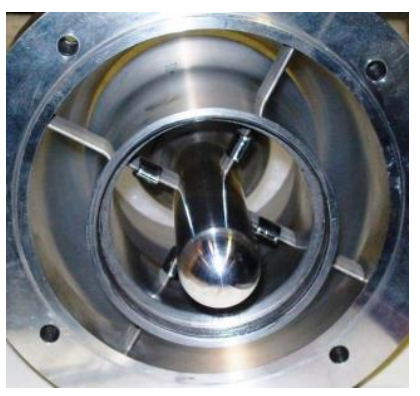

(b)

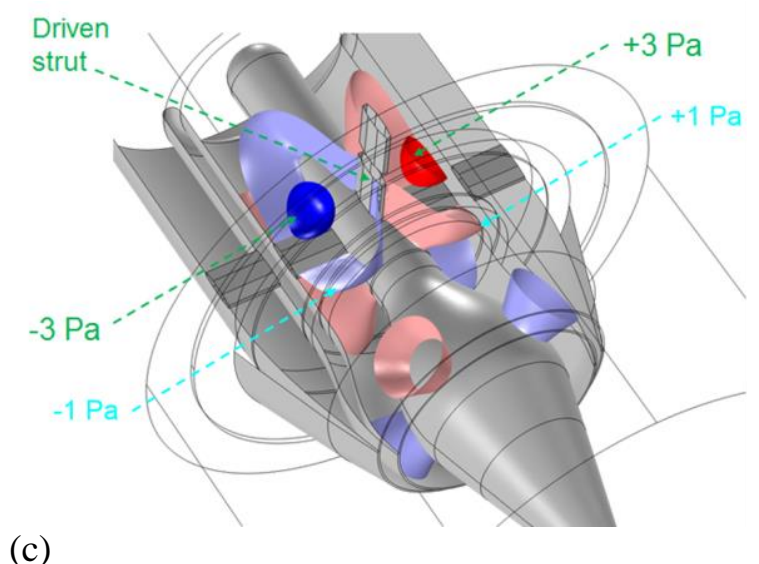

Fig. 1 Reproduced from Ref. [1]. (a) Drawing of the 2-stream model-scale nozzle, (b) nozzle viewed from upstream, here the struts are fitted with semi-span caps, (c) numerical result showing four iso-surfaces of pressure $( \pm 3$ and $\pm 1 \mathrm{~Pa})$ at a resonant frequency of $4.5 \mathrm{kHz}$.

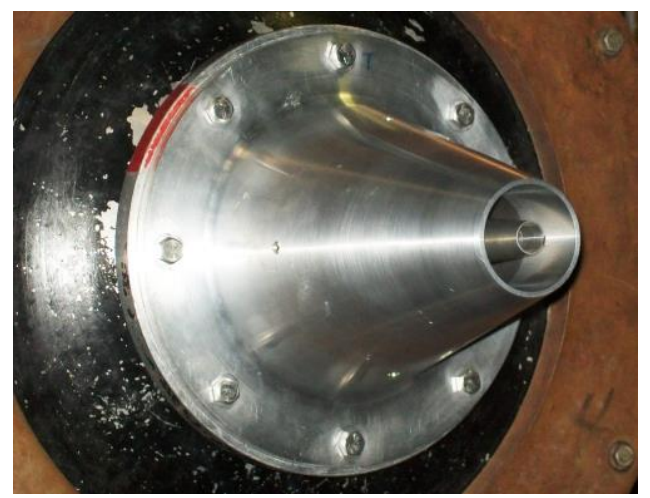

(a)

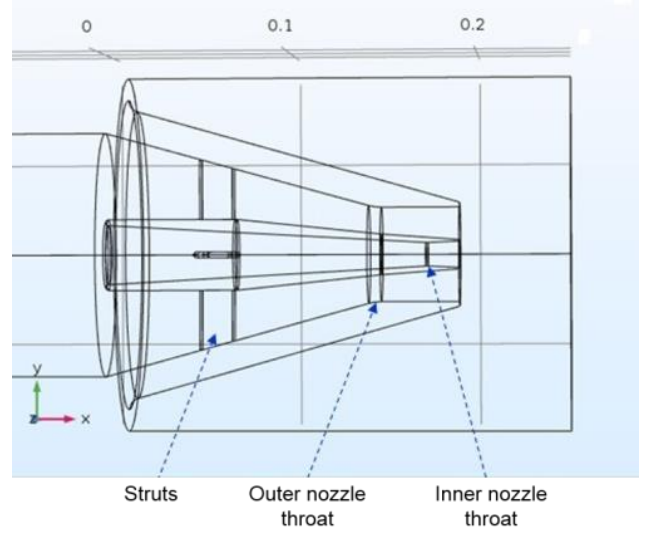

(b)

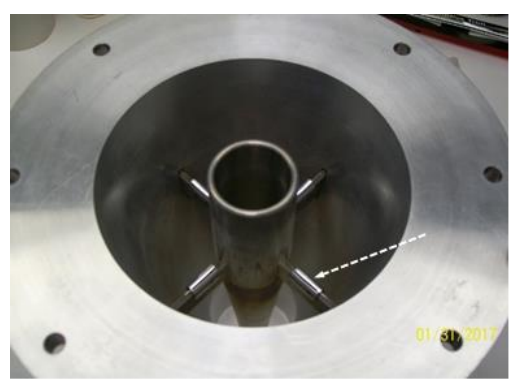

(c)

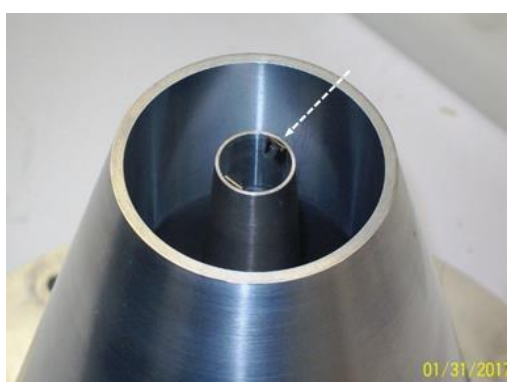

(d)

Fig 2 (Continued next column) 


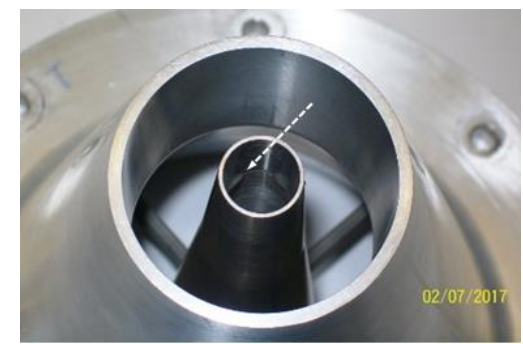

(e)

Fig. 2 Experimental facility. (a) Nozzle mounted on jet facility, (b) sketch of interior, (c) Rear view of nozzle with caps on struts, (d) two tabs on inner nozzle and (e) epoxy beads near the throat of inner nozzle. White arrows in the pictures point to a cap, tab or the epoxy bead.

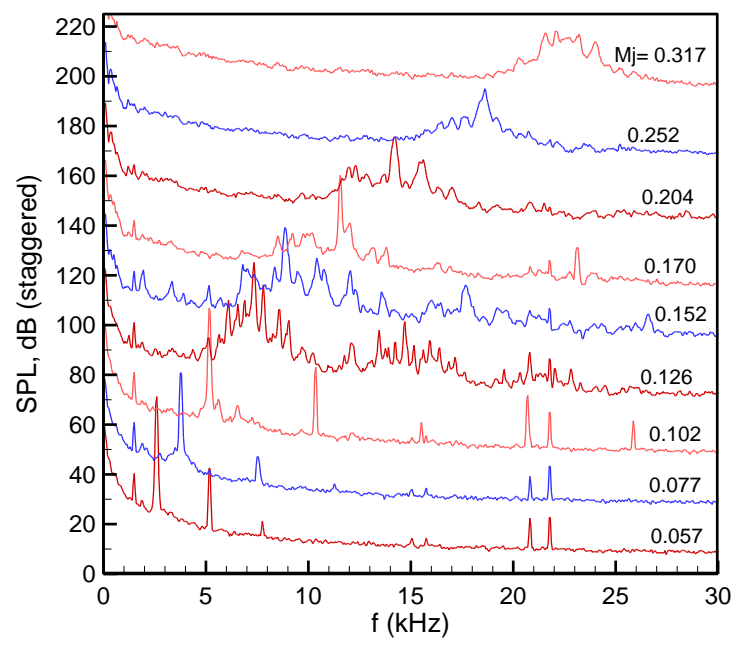

Fig. 3 Sound pressure level (SPL) spectra at different jet Mach number $\left(M_{j}\right)$.

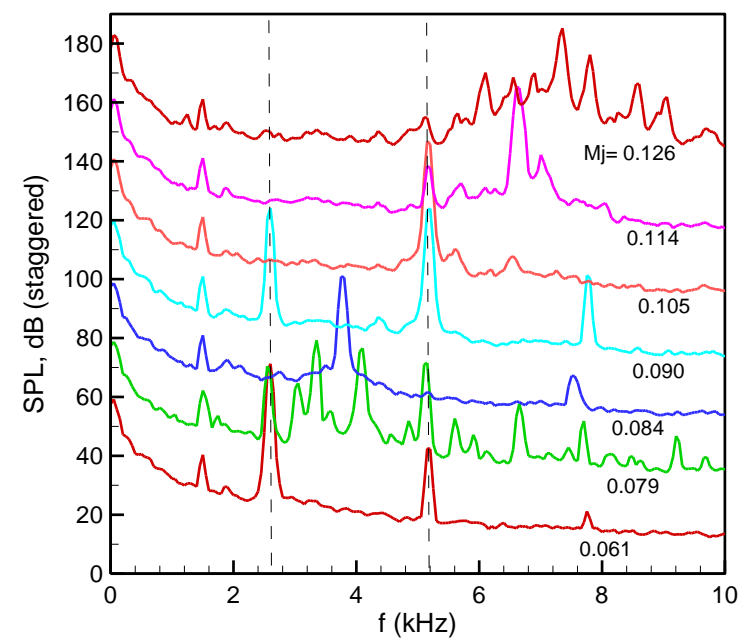

Fig. 4 SPL spectra in low $M_{j}$-range with expanded abscissa. (a)

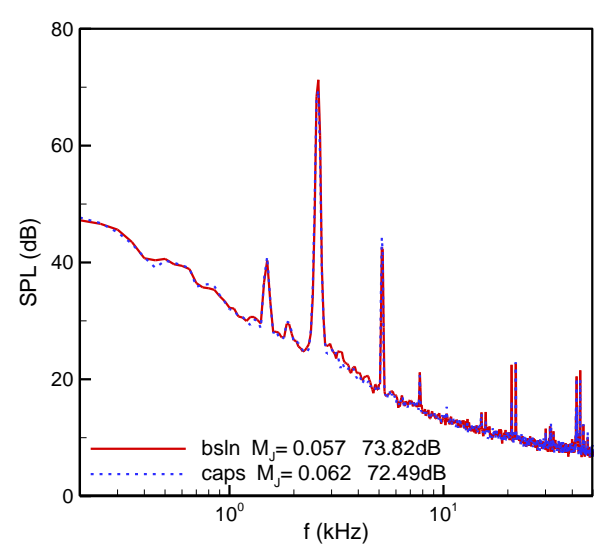

(b)

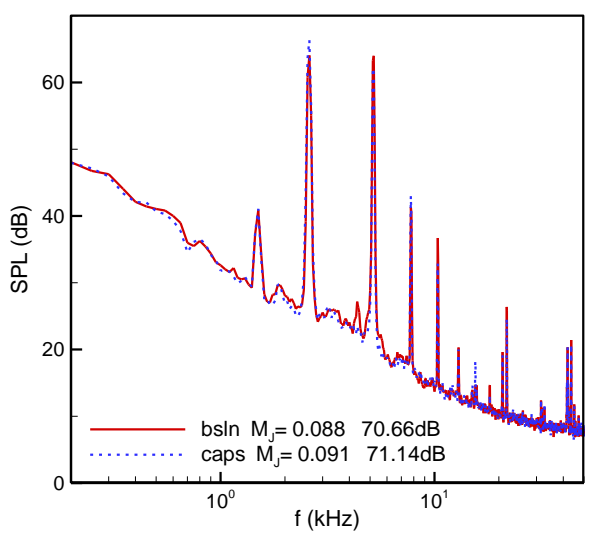

(c)

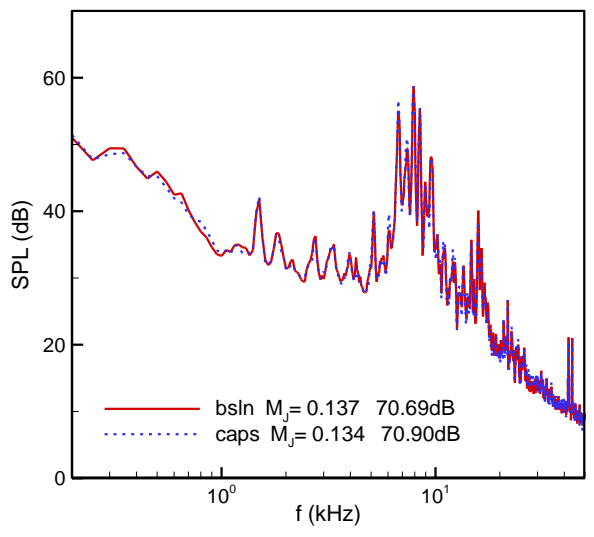

Fig. 5 Effect of caps on struts (Fig. 2c). Three sets of SPL spectra in (a), (b) and (c) are for $M_{j}=$ $0.061,0.09$ and 0.137 , respectively. Red line for baseline ('bsln') case without caps, blue dotted line for caps. 


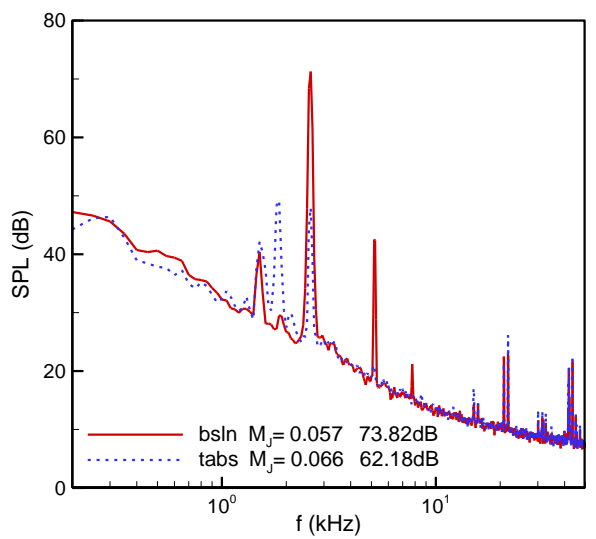

(a)

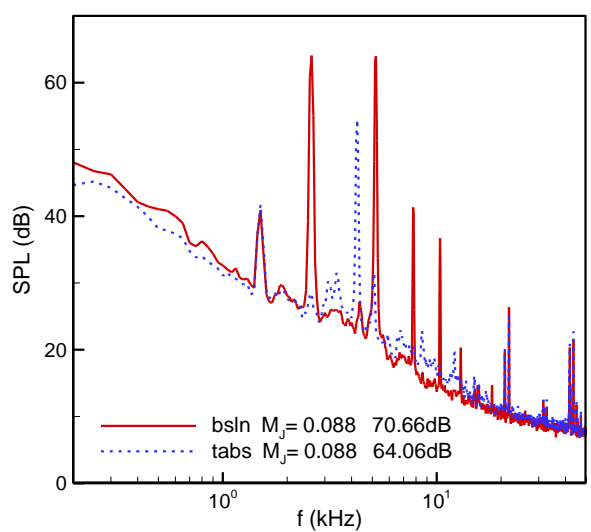

(b)

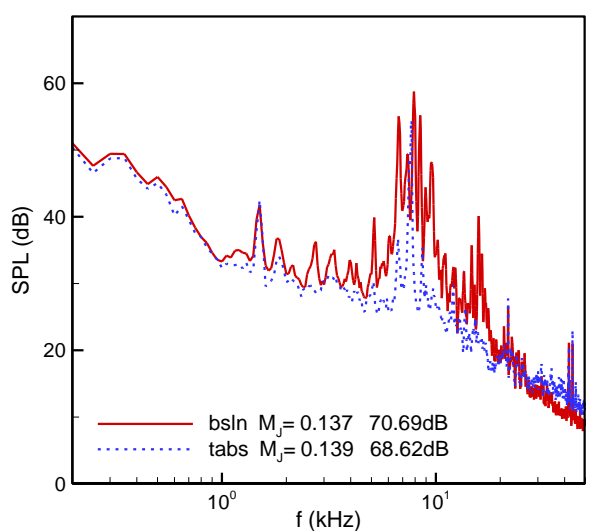

(c)

Fig. 6 Effect of tabs on inner nozzle (Fig. 2d), for the same nominal jet Mach numbers as in Fig. 5.

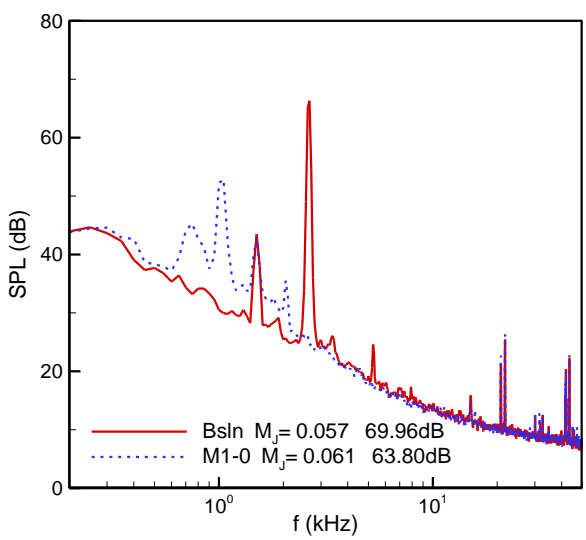

(a)

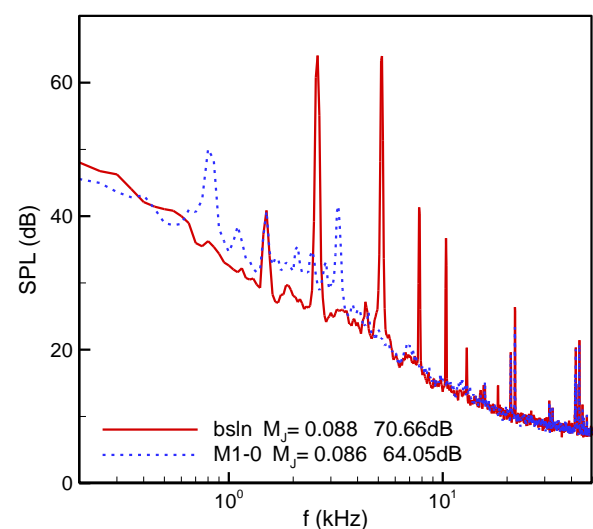

(b)

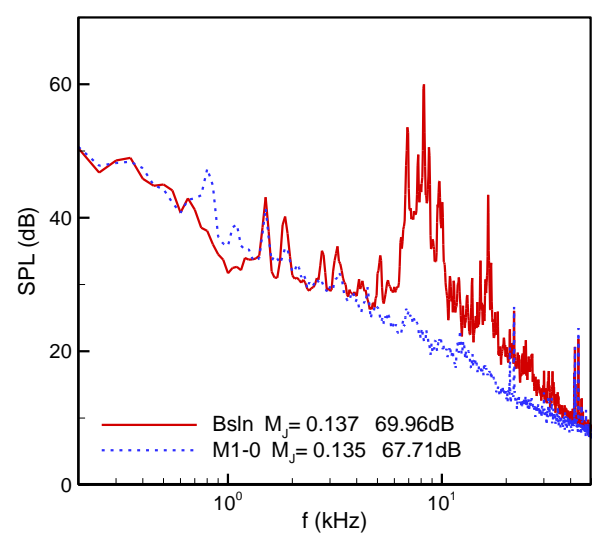

(c)

Fig. 7 Effect of blocking flow through the inner nozzle, for the same nominal jet Mach numbers as in Fig. 5. 


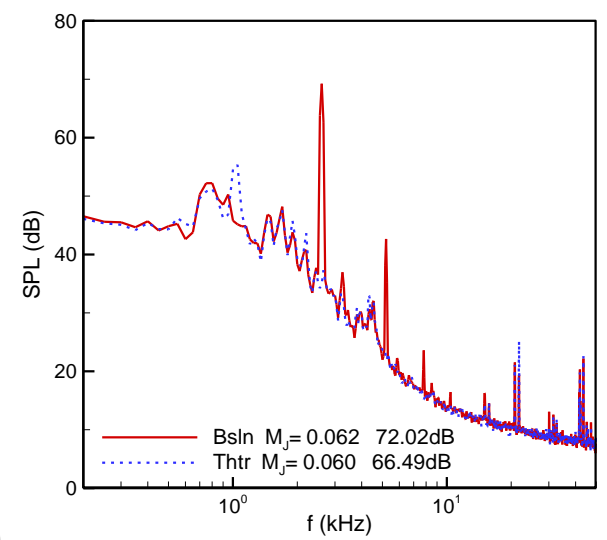

(a)

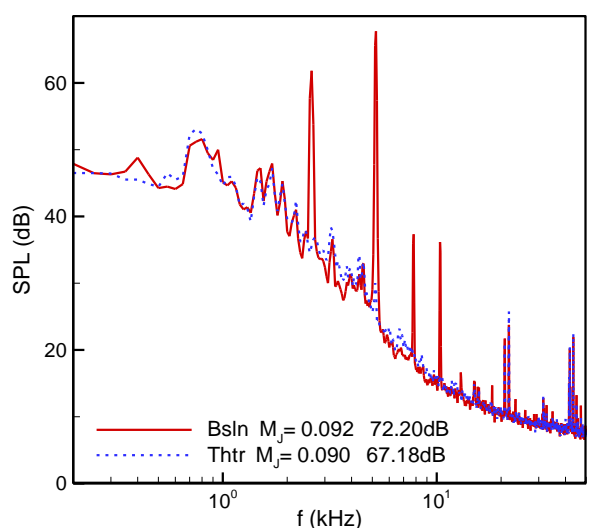

(b)

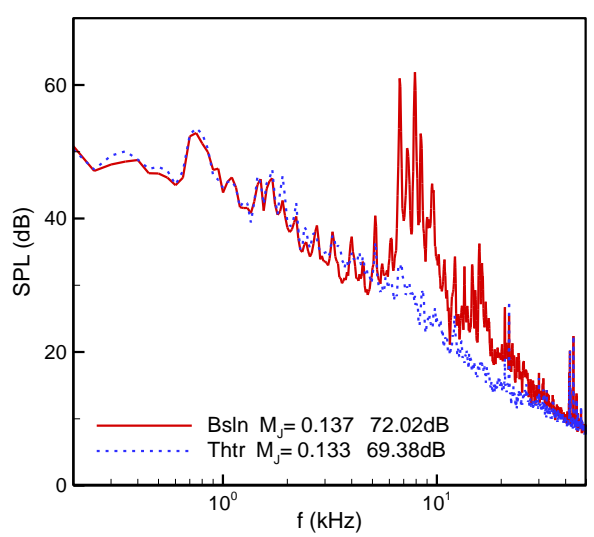

(c)

Fig. 8 Effect of epoxy beads near throat of inner nozzle, for the same nominal jet Mach numbers as in Fig. 5.

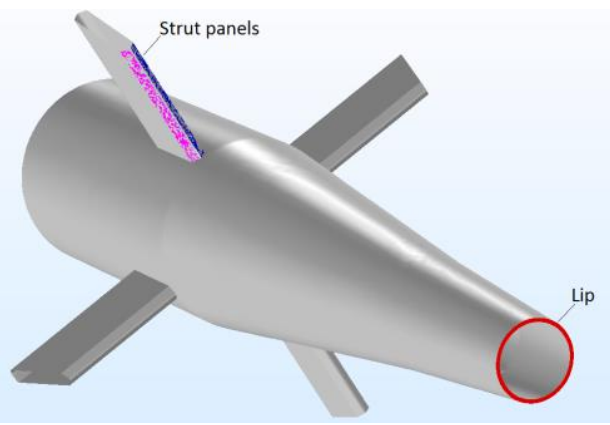

Fig. 9 Drawing of inner nozzle and struts illustrating excitation method in numerical simulation. 'Inner lip': perturbation at the inner nozzle lip with $\pm 1 \mathrm{~Pa}$; 'Strut panels': perturbation on two panels near trailing edge of one strut with $\pm 1 \mathrm{~Pa}$.

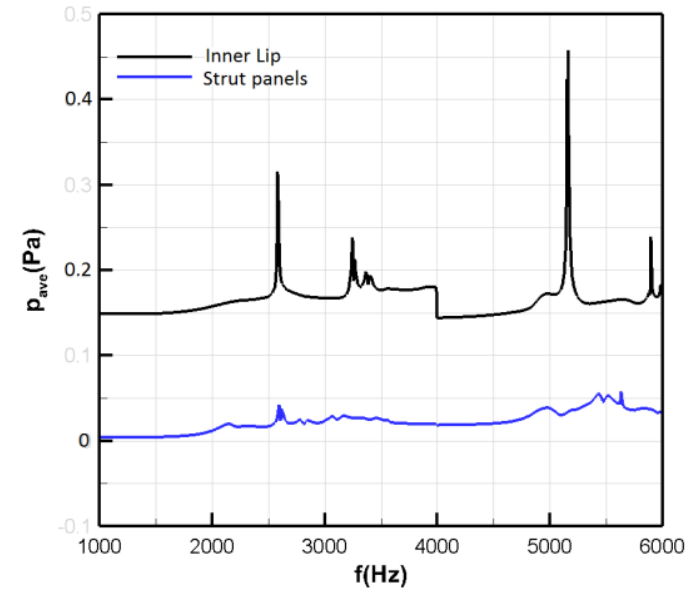

Fig. 10 Spectrum of response function (average of absolute pressure). Black line: 'Inner Lip' excitation, blue line: 'strut panel' excitation (Fig. 9). 


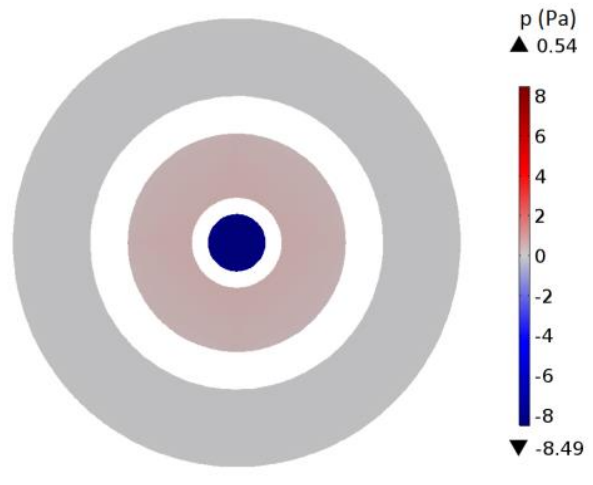

Fig. 11 Pressure distribution on a cross-section just downstream of the struts at a given phase of the 'fundamental' spectral peak $(2579 \mathrm{~Hz}$; Fig. 10); inner lip excitation.

(a)
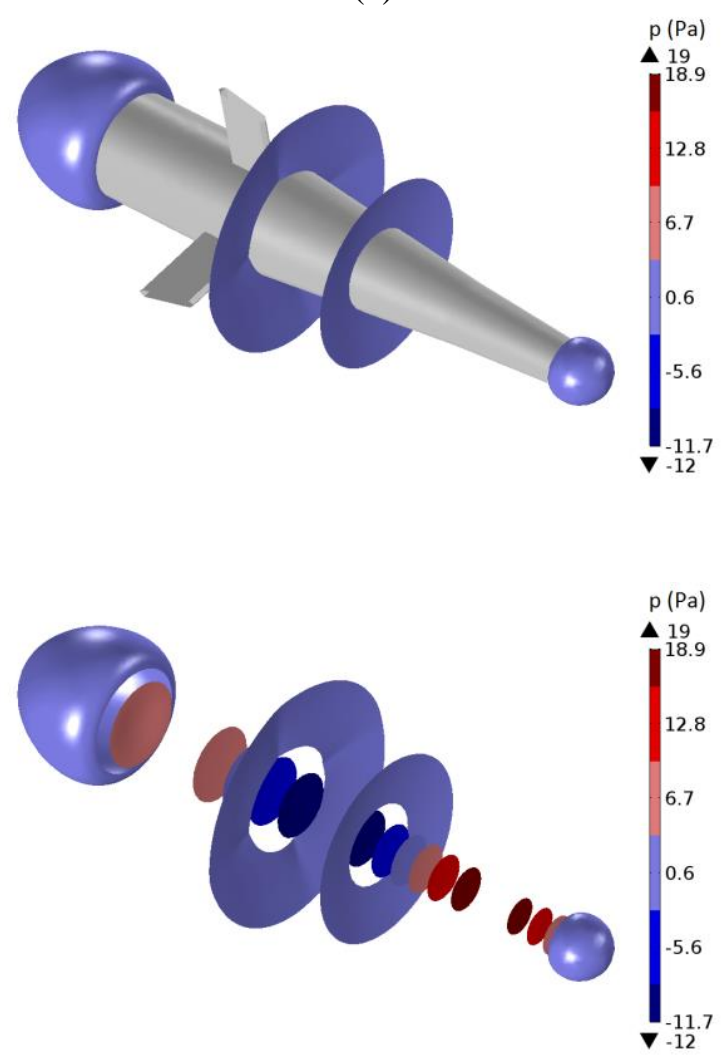

(b)

Fig. 12 Iso-surfaces of instantaneous pressure corresponding to the fundamental spectral peak (2579 $\mathrm{Hz}$ ). (a) Distribution shown with outer nozzle removed, (b) same distribution with inner nozzle also removed.

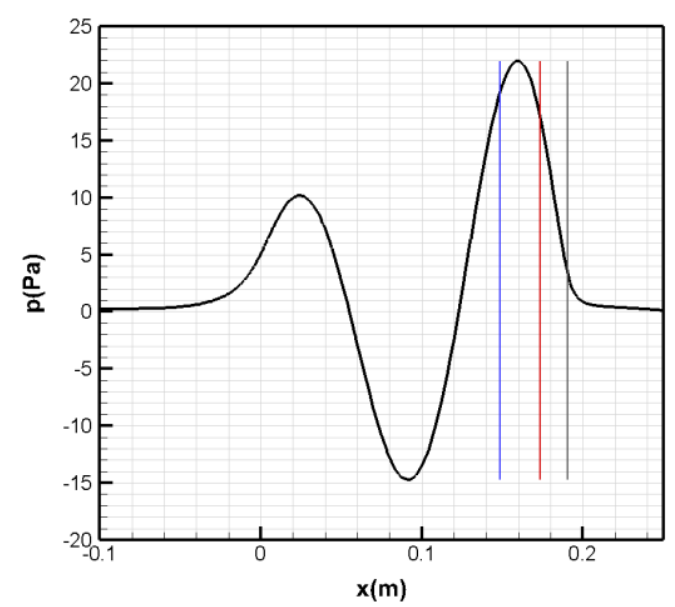

Fig. 13 Pressure distribution at a given phase along centerline corresponding to the fundamental spectral peak $(2579 \mathrm{~Hz})$. The nozzle exit, the inner nozzle throat and the outer nozzle throat locations are shown by the vertical lines.

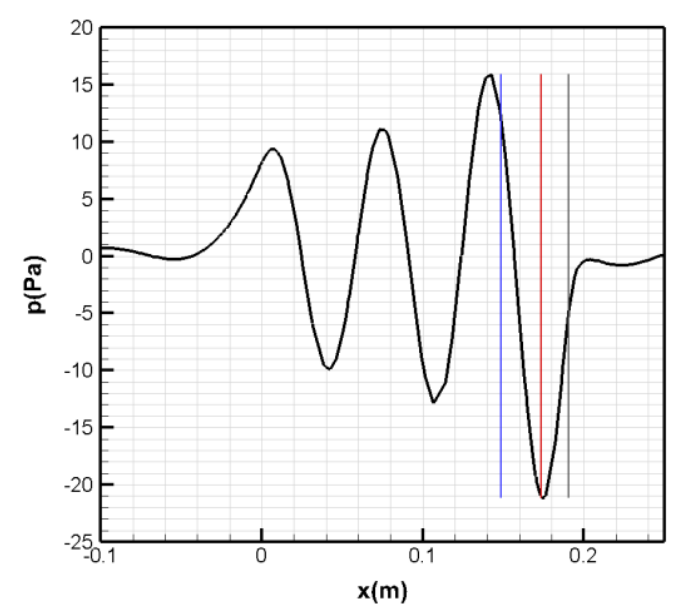

Fig. 14 Pressure distribution at a given phase along centerline corresponding to the spectral peak at $5200 \mathrm{~Hz}$ shown similarly as in Fig. 13. 